\title{
Glassy behavior of molecular crystals: A comparison between results from MD-simulation and mode coupling theory
}

\author{
M. Ricker ${ }^{a}$, F. Affouard ${ }^{b}$, R. Schilling ${ }^{a}$ and M. Descamps ${ }^{b}$ \\ ${ }^{a}$ Institut für Physik, Johannes Gutenberg-Universität Mainz, Staudinger Weg 7, D-55099 \\ Mainz, Germany \\ ${ }^{\mathrm{b}}$ Laboratoire de Dynamique et Structure des Matériaux Moléculaires, UMR CNRS 8024, \\ Université Lille I, 59655 Villeneuve d'Ascq Cedex France
}

\begin{abstract}
We have investigated the glassy behavior of a molecular crystal built up with chloroadamantane molecules. For a simple model of this molecule and a rigid fcc lattice a MD simulation was performed from which we obtained the dynamical orientational correlators $S_{\lambda \lambda^{\prime}}(\mathbf{q}, t)$ and the "self" correlators $S_{\lambda \lambda^{\prime}}^{(s)}(t)$, with $\lambda=(\ell, m), \lambda^{\prime}=\left(\ell^{\prime}, m^{\prime}\right)$. Our investigations are for the diagonal correlators $\lambda=\lambda^{\prime}$. Since the lattice constant decreases with decreasing temperature which leads to an increase of the steric hindrance of the molecules, we find a strong slowing down of the relaxation. It has a high sensitivity on $\lambda, \lambda^{\prime}$. For most $(\ell, m)$, there is a two-step relaxation process, but practically not for $(\ell, m)=(2,1),(3,2),(4,1)$ and $(4,3)$. Our results are consistent with the $\alpha$-relaxation scaling laws predicted by mode coupling theory from which we deduce the glass transition temperature $T_{c}^{M D} \cong 217 K$. From a first principle solution of the mode coupling equations we find $T_{c}^{M C T} \cong 267 \mathrm{~K}$. Furthermore mode coupling theory reproduces the absence of a two-step relaxation process for $(\ell, m)=(2,1),(3,2),(4,1)$ and $(4,3)$, but underestimates the critical nonergodicity parameters by about 50 per cent for all other $(\ell, m)$. It is suggested that this underestimation originates from the anisotropic crystal field which is not accounted for by mode coupling theory. Our results also imply that phonons have no essential influence on the long time relaxation.
\end{abstract}

Key words: PACS numbers: 64.70.Pf, 61.43.-j

\section{Introduction}

The mode coupling theory (MCT) [1] is the most successful microscopic approach to describe glassy dynamics of supercooled liquids on a qualitative, and partly even on a quantitative level [2,3,4]. Particularly, first-principle comparisons between the 
solutions of MCT equations and results from experiments and simulations for various systems like the binary Lennard-Jones liquid [5], liquids of diatomic molecules [6,7], water [8,9], silica melt [10] and a model for orthoterphenyl [11] have confirmed the quality of MCT. These comparisons were restricted to the glass order parameters, the nonergodicity parameters. Going beyond that, even time dependent quantities, like the intermediate scattering function, have been compared with each other for the binary Lennard-Jones liquid [12], binary mixtures of hard spheres $[13,14]$ and the polydisperse quasi-hard-sphere system [15], again demonstrating consistency with MCT.

Glassy dynamics of systems with self-generated disorder is not restricted to liquids. Molecular crystals in their plastic phase exhibit glassy behavior as well. This has been found experimentally three decades ago [16]. Recently two of the present authors have extended MCT to molecular crystals [17]. Using the static structure factors from Percus-Yevick theory [18] the orientational glass transition of uniaxial hard ellipsoids on a simple cubic lattice has been investigated [17]. There it has been found that this transition is not driven by an orientational cage effect, analogous to supercooled liquids, but by the growth of orientational order. This growth manifests itself in an increase of the static orientational correlators at the Brillouin center or edge, which in turn leads to an increase of the memory kernel. However, before the corresponding orientational correlation length diverges at the corresponding equilibrium phase transition line, the nonlinear feedback mechanism of MCT results in an orientational glass transition. The same mechanism has been identified for a liquid of uniaxial hard ellipsoids [19]. Accordingly, the MCT glass transition line is located within the orientationally disordered ergodic phase $[17,19]$ and not in the supercooled regime.

One may ask whether there also exists a cage-effect-driven glass transition for molecular crystals. Good candidates are plastic crystals which undergo a first order equilibrium phase transition to an orientationally ordered phase. Several such systems exist such as cyanoadamantane, ethanol, cyclooctanol, difluorotetrachloroethane or $\mathrm{C}_{60}$ [20]. Another one is chloroadamantane, which exhibits an equilibrium phase transition at $T_{e q}^{e x p} \cong 244 K$ [21]. A simple model for chloroadamantane (see next section) has been studied by MD simulations [22,23]. A MCT analysis for the critical amplitudes and the $\alpha$-relaxation time have demonstrated consistency with MCT predictions [23]. These predictions can really be extended from liquids to plastic crystals because the MCT equations for the latter have the same mathematical structure than for multi-component simple liquids [17].

It is the main goal of the present paper to perform a first principle comparison between the results for chloroadamantane from a MD-Simulation and MCT. The outline of our contribution is as follows. In the second section we will describe the model, introduce the orientational correlation functions, give some details of the simulational procedure and shortly describe the relevant MCT equations. Results are presented and discussed in the third section and the final section contains a 
summary and some conclusions.

\section{Model and technical details}

Chloroadamantane $\mathrm{C}_{10} \mathrm{H}_{15} \mathrm{Cl}$ is a rather huge molecule which belongs to the substituted adamantane family. It shows a plastic phase structure isomorphous to cyanoadamantane, but the chloroadamantane molecule possesses a smaller substitute and a faster dynamics well adapted for MD simulation investigations. Chloroadamantane undergoes at $T \simeq 244 K$ a first order transition from an ordered monoclinic structure to a rotator phase with face-centered-cubic (fcc) symmetry [21]. The plastic-liquid transition occurs at $T_{m} \simeq 442 \mathrm{~K}$ [21].

Table 1

Parameters for the two-site chloroadamantane model.

\begin{tabular}{|l|c|c|c|c|}
\hline Site-Site & $\mathrm{p}$ & $\mathrm{q}$ & $\epsilon(\mathrm{kJ} / \mathrm{mol})$ & $\sigma(\AA)$ \\
\hline $\mathrm{Cl}-\mathrm{Cl}$ & 12 & 6 & 1.441 & 3.350 \\
\hline $\mathrm{Cl}-\mathrm{Adm}$ & 14 & 8 & 3.087 & 4.786 \\
\hline $\mathrm{Adm}-\mathrm{Adm}$ & 16 & 11 & 12.47 & 6.200 \\
\hline
\end{tabular}

The simulated system is composed of rigid linear molecules with two sites: one chlorine atom (noted $\mathrm{Cl}$ ) and one super atom (noted Adm) that models the adamantane part $\mathrm{C}_{10} \mathrm{H}_{15}$. The moment of inertia is $302.733 \mathrm{amu} . \AA^{2}$. Molecular dynamics calculations were performed on a system of $N=256$ molecules $(4 \times 4 \times 4 \mathrm{fcc}$ crystalline cells) interacting through a Lennard-Jones short range site-site potential of the form

$$
v(r)=4 \epsilon\left((\sigma / r)^{p}-(\sigma / r)^{q}\right)
$$

where $r$ is the distance between two different sites. The parameters $\epsilon, \sigma, p$ and $q$ are specified in table 1 .

The chloroadamantane molecule possesses a relatively large dipolar moment $\vec{\mu}$ (2.39 Debyes) which is parallel to the molecular axis. The electrostatic interactions were handled by the Ewald method with two partial charges $(q= \pm 0.151 e)$ localized on both sites. Newton's equations of motion were solved with a time step of $\Delta t=5 \mathrm{fs}$. We worked in the NPT (constant number of molecules, temperature and pressure) and NVT (constant number of molecules, temperature and volume) statistical ensembles with periodic boundaries conditions. The sample was first equilibrated in the NPT ensemble. Then, MD runs at constant volume using the average volume determined from the NPT simulations were performed. 


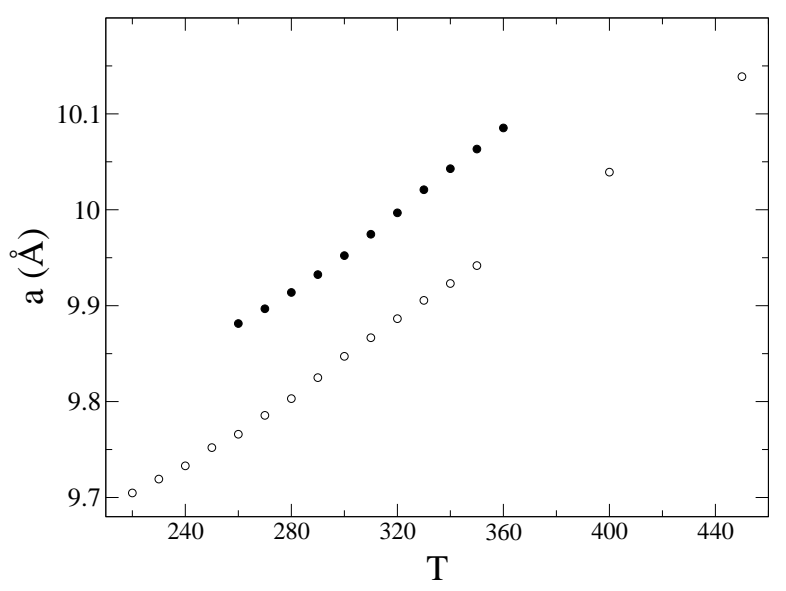

Fig. 1. Temperature dependence of the lattice constant obtained from experiments (full circles) and MD-simulation (open circles)

Since our molecule-model has one rotational symmetry axis we can use the angles $\Omega_{n}(t)=\left(\Theta_{n}(t), \phi_{n}(t)\right)$ to characterize the orientation of the $\mathrm{n}$-th molecule at site $n$ and time $t$. The microscopic local orientational density is given by:

$$
\rho_{n}(\Omega, t)=\delta\left(\Omega \mid \Omega_{n}(t)\right)
$$

with $\delta\left(\Omega \mid \Omega^{\prime}\right)=(\sin \Theta)^{-1} \delta\left(\Theta-\Theta^{\prime}\right) \delta\left(\phi-\phi^{\prime}\right)$. Expansion with respect to spherical harmonics $Y_{\lambda}(\theta, \phi), \lambda=(\ell, m)$ and performing a lattice Fourier transform leads to the tensorial orientational density modes:

$$
\rho_{\lambda}(\mathbf{q}, t)=i^{l} \sum_{n=1}^{N} Y_{\lambda}\left(\Omega_{n}(t)\right) e^{i \mathbf{q} \mathbf{R}_{n}}
$$

where $N$ is the number of lattice sites and $\mathbf{R}_{\mathbf{n}}$ the lattice vector of the $n$th lattice site. The reader should note that we assume a rigid lattice and that $\mathbf{q}$ is restricted to the 1. Brillouin zone. Introducing the fluctuations $\delta \rho_{\lambda}(\mathbf{q}, t)=\rho_{\lambda}(\mathbf{q}, t)-\left\langle\rho_{\lambda}(\mathbf{q}, t)\right\rangle$ we can define the time dependent, tensorial orientational correlators:

$$
S_{\lambda \lambda^{\prime}}(\mathbf{q}, t)=\frac{4 \pi}{N}\left\langle\delta \rho_{\lambda}^{*}(\mathbf{q}, t) \delta \rho_{\lambda^{\prime}}(\mathbf{q}, 0)\right\rangle
$$

where $\langle\ldots\rangle$ denotes the canonical average over the initial conditions. Note that the absence of a head-tail symmetry leads to nontrivial correlators for all $\ell \geq 1$, while the absence of phonons leads to $S_{\lambda \lambda^{\prime}}(\mathbf{q}, t)=0$ for $\lambda=(0,0)$ and/or $\lambda^{\prime}=(0,0)$ within the 1 . Brillouin zone.

These correlators have been investigated by a MD-simulation. In contrast to earlier work $[22,23]$ the present simulation has been done for each temperature $T$ for a rigid lattice with lattice constant $a(T)$ determined from the average size of the system obtained from NPT simulations. It is represented in Figure 1, which also 


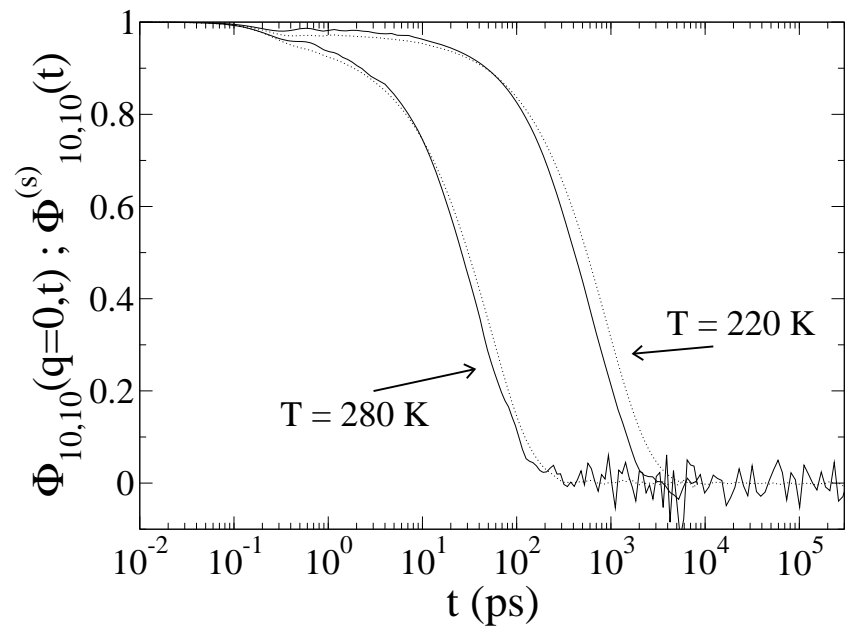

Fig. 2. Time-dependence of the collective correlator $(\mathbf{q}=\mathbf{0})$ (solid line) and "self" correlator (dotted line) for $\lambda=\lambda^{\prime}=(1,0)$ and $T=220 K, 280 K$

contains the experimental result [21]. It is the shrinking of the lattice constant (cf. Fig. 1) which leads to an increase of the steric hindrance with decreasing temperature and in turn to a slowing down of the orientational dynamics.

On the other hand, MCT provides an equation of motion for $S_{\lambda \lambda^{\prime}}(\mathbf{q}, t)$ which requires the static correlators $S_{\lambda \lambda^{\prime}}(\mathbf{q}, 0)$ as an input. Because of the crystal's anisotropy, these correlators also depend on the direction of $q$. Therefore the numerical solution of the $\mathrm{t}$-dependent MCT equations is rather involved such that we will restrict ourselves to the determination of the MCT glass transition temperature $T_{c}$ and the normalized nonergodicity parameters

$$
\begin{array}{r}
f_{\lambda \lambda^{\prime}}(\mathbf{q})=\lim _{t \rightarrow \infty} \phi_{\lambda \lambda^{\prime}}(\mathbf{q}, t), \\
\phi_{\lambda \lambda^{\prime}}(\mathbf{q}, t)=S_{\lambda \lambda^{\prime}}(\mathbf{q}, t) /\left[S_{\lambda \lambda}(\mathbf{q}, 0) S_{\lambda^{\prime} \lambda^{\prime}}(\mathbf{q}, 0)\right]^{1 / 2}
\end{array}
$$

taken at $T=T_{c}$. They are solutions of an infinite set of nonlinear coupled algebraic equations [17]:

$$
f_{\lambda \lambda^{\prime}}(\mathbf{q})=\mathcal{T}_{\lambda \lambda^{\prime}}\left(\mathbf{q},\left\{f_{\lambda \lambda^{\prime}}(\mathbf{q})\right\}\right) .
$$

The mode coupling polynomial $\mathcal{T}_{\lambda \lambda^{\prime}}$ is related to the memory kernel and depends on temperature through the static correlators. The static correlators have been taken from the MD-simulation. The numerical solution of Eq. (5) requires a truncation at $\ell_{\text {max }}$, for which we have chosen $\ell_{\max }=4$.

\section{Results}

As mentioned above the collective correlators (Eq. (3)) are nontrivial for $\ell \geq 1, \ell^{\prime} \geq$ 1. Besides these, one can also determine the "self" correlators: 

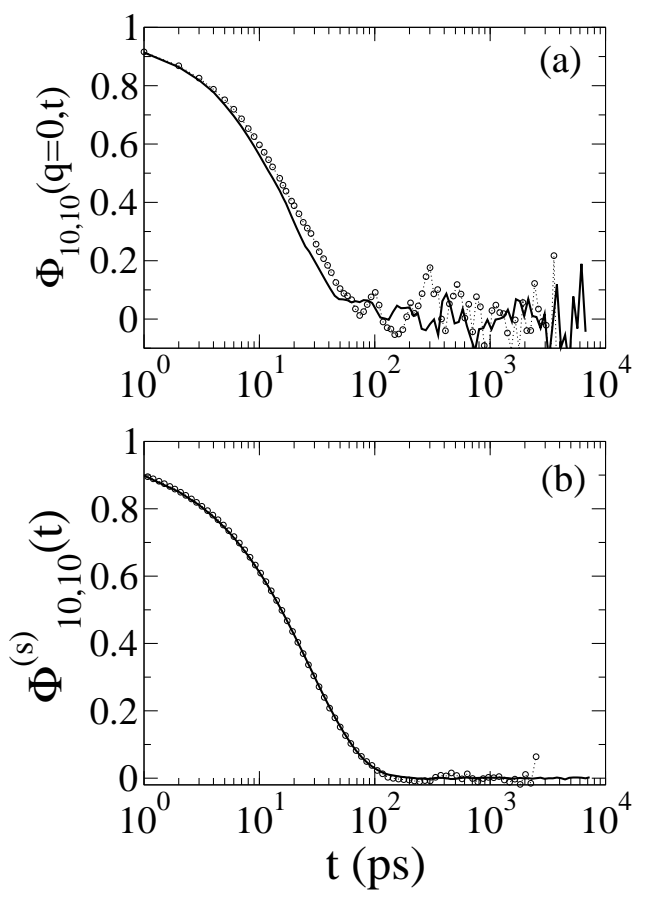

Fig. 3. Time-dependence of (a) the collective correlator $(\mathbf{q}=\mathbf{0})$ and (b) "self" correlator for $\lambda=\lambda^{\prime}=(1,0)$ at $T=300 K$ for $N=256$ particles (open circles) and $N=2048$ particles (solid line), respectively.

$$
\begin{aligned}
S_{\lambda \lambda^{\prime}}^{(s)}(t) & =\frac{4 \pi}{N} \sum_{n=1}^{N}\left\langle\delta \rho_{n, \lambda}^{*}(t) \delta \rho_{n, \lambda^{\prime}}(0)\right\rangle \\
& =\frac{1}{N} \sum_{\mathbf{q} \in 1 . B . Z} S_{\lambda \lambda^{\prime}}(\mathbf{q}, t)
\end{aligned}
$$

$\delta \rho_{n, \lambda}(t)$ is the expansion coefficient of $\delta \rho_{n}(\Omega, t)$ with respect to $Y_{\lambda}(\Omega)$. Figure 2 shows the normalized collective correlator at $\mathbf{q}=\mathbf{0}$ and the "self" correlator for $\lambda=\lambda^{\prime}=(1,0)$ and $T=220 K$ and $280 K$. First of all we observe a slowing down of the $\alpha$-relaxation by a factor of about twenty by changing the temperature from $280 K$ to $220 K$, i.e. by about one fifth. Second, no significant difference occurs between the collective and the "self" correlator. The former possesses some oscillations on the microscopic time scale below $10 \mathrm{ps,} \mathrm{in} \mathrm{contrast} \mathrm{to} \mathrm{the} \mathrm{self} \mathrm{correlator.}$ At the lowest temperature $220 K$ both reveal a two-step relaxation process, however, with a plateau height very close to one. Since the system size of 256 sites is not very huge, we have also performed a simulation at $T=300 K$ with 2048 sites. The result for $\lambda=\lambda^{\prime}=(1,0)$ is presented in Figure $3 \mathrm{a}$ (collective) and Figure $3 \mathrm{~b}$ ("self") and shows no significant finite size effects. Whether this holds also at the lowest temperature $T=220 \mathrm{~K}$ has not been possible to study because of very long equilibration times.

One of the predictions of MCT is the validity of the $t-T$ superposition principle, 

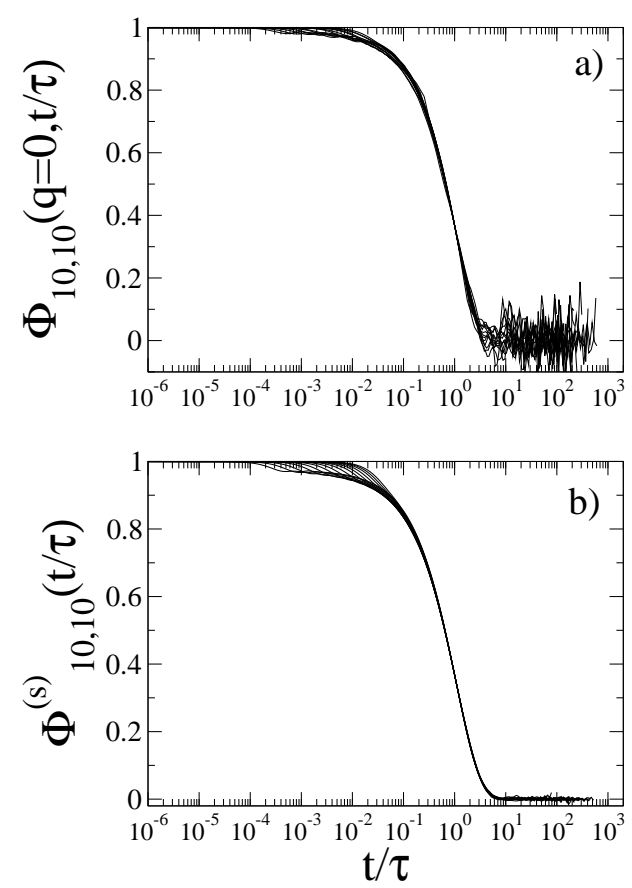

Fig. 4. (a) Collective correlator $(\mathbf{q}=\mathbf{0})$ and (b) "self" correlator for $\lambda=\lambda^{\prime}=(1,0)$ versus the rescaled time $t / \tau$. $\tau$ has been determined as the time at which the correlators have decayed to $1 / e$. Correlators are shown for $T=220 K$ to $350 K$ in $10 K$ steps.

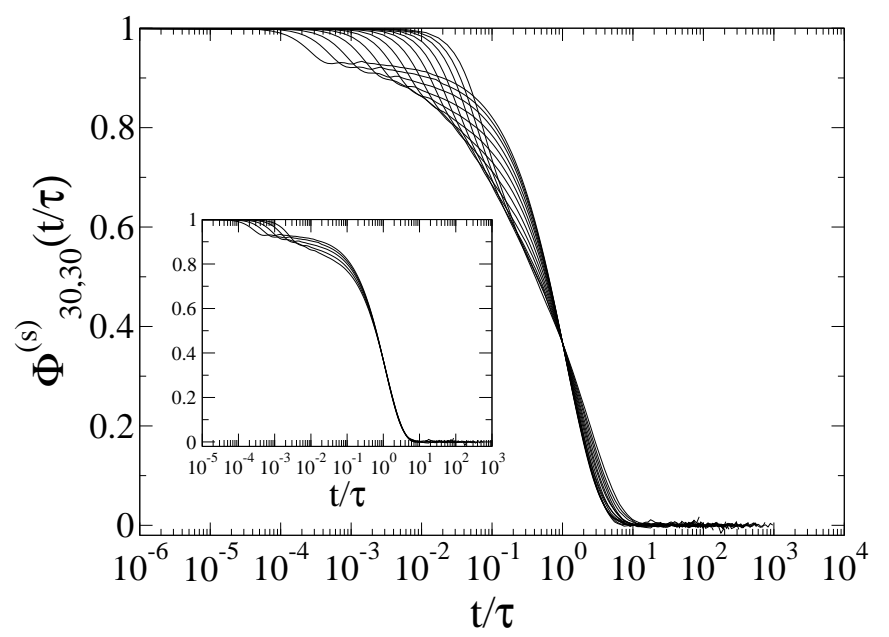

Fig. 5. Same as Fig.4b, but for $\lambda=\lambda^{\prime}=(3,0)$. The inset shows the lowest five temperatures, only.

i.e. scaling of t by the $\alpha$-relaxation time $\tau_{\lambda \lambda^{\prime}}(\mathbf{q}, T)$ should yield a data collapse for all $T$ and $t$ large enough. Taking $\tau_{\lambda \lambda^{\prime}}(\mathbf{q}, T)$ as the time at which $\phi_{\lambda \lambda^{\prime}}(\mathbf{q}, t)$ has decayed to $1 / e$ the rescaled data are given in Figure $4 \mathrm{a}$ and Figure $4 \mathrm{~b}$ for the collective $(\mathbf{q}=\mathbf{0})$ and "self" correlator, respectively, for $\lambda=\lambda^{\prime}=(1,0)$. A satisfactory collapse is found over a rather huge temperature range. As can be seen 

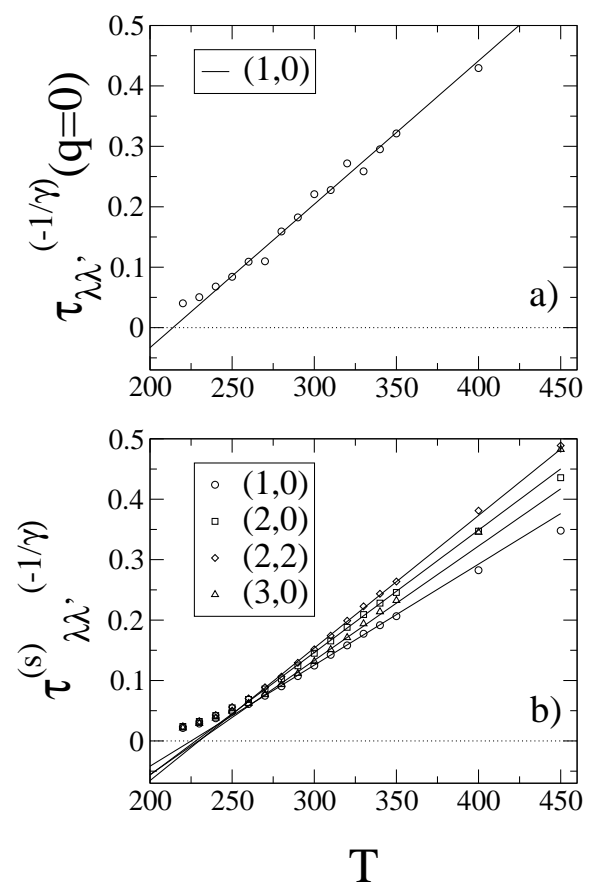

Fig. 6. $\tau^{-1 / \gamma}$ versus $T$ for (a) the collective correlator for $\lambda=\lambda^{\prime}=(1,0)$ at $\mathbf{q}=\mathbf{0}$ and (b) the "self" correlator for $\lambda=\lambda^{\prime}=(1,0),(2,0),(2,2)$ and $(3,0)$.

from Figure 5 this is not true for $\phi_{30,30}^{(s)}(t)$. Restriction to $220 K \leq T \leq 260 K$ leads to a satisfactory result for $t / \tau>1$, which, however, excludes the von Schweidler regime (see inset of Fig. 5). One of the highly nontrivial predictions of MCT is the validity of the power law dependence of $\tau$ on $\left(T-T_{c}\right)$ :

$$
\tau(T) \sim\left(T-T_{c}\right)^{-\gamma} \quad, \quad T \geq T_{c}
$$

Figure 6b shows $\tau^{-1 / \gamma}$ versus $T$ for the "self" correlators for different $\lambda=\lambda^{\prime}$ and Figure 6a the corresponding result for the collective one at $\mathbf{q}=\mathbf{0}$ and $\lambda=\lambda^{\prime}=$ $(1,0)$. There is a linear behavior for $250 K<T<350 K$. Its extrapolation to zero yields

$$
T_{c}^{M D} \cong 217 \pm 5 K
$$

For the exponent we find $\gamma_{\lambda \lambda^{\prime}} \cong 2.0 \pm 0.2$ depending on $\lambda=\lambda^{\prime}$.

An interesting behavior has been found for the collective correlators with $\lambda=\lambda^{\prime}$ and $\ell=2,3$. The corresponding results at the Brillouin center are presented in Figure $7 \mathrm{a}$ and $7 \mathrm{~b}$ for the lowest temperature $T=220 \mathrm{~K}$. Both correlators exhibit a high sensitivity on the $m$-index. For $\ell=2$ and $\ell=3$ there is a two-step relaxation for $m=0,2$ and $m=0,1,3$, respectively, with rather high plateau values, particularly for $\ell=2$. But for $\ell=2, m=1$ and $\ell=3, m=2$ there is a rather unusual t-dependence showing no typical two-step relaxation with a convex "short" and a concave "long" time part joining at an inflection point (the $\beta$-relaxation regime of 

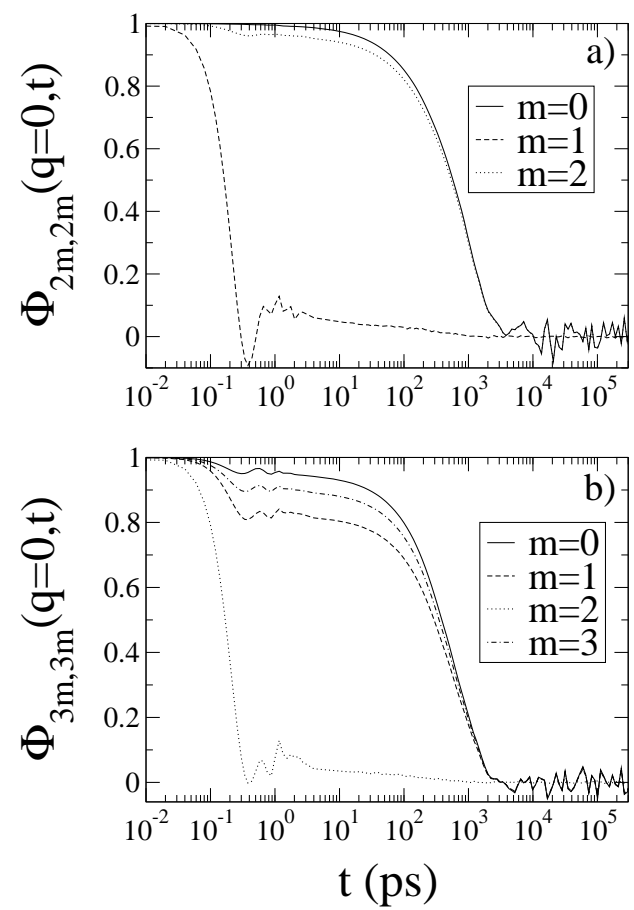

Fig. 7. Time- and m-dependence of the collective correlator at $\mathbf{q}=\mathbf{0}$ and $T=220 \mathrm{~K}$ for (a) $\ell=\ell^{\prime}=2$ and (b) $\ell=\ell^{\prime}=3$

MCT). The "long" time relaxation for $t>4 p s$ has a convex curvature on the logarithmic time scale starting at a height of about 0.05 for both cases. If this height is interpreted as a plateau height, it has an unusual small value. Surprisingly, it is observed that the correlators without two step relaxation decay faster as the temperature is lowered.

For $(\ell, m)=(4,0),(4,2)$ and $(4,4)$ we also have a two step relaxation, while for $(\ell, m)=(4,1)$ and $(4,3)$ it is missing, as for $(2,1),(3,2)$. The results for $\ell=4$ yield no new physical insight and are not shown in this work.

In a final step we have investigated how far these plateau values (which are roughly equal to the critical nonergodicity parameters) are reproduced by MCT for plastic crystals. First, we have fitted the MD-results by the von Schweidler law in order to determine the critical nonergodicity parameters $f_{\lambda \lambda^{\prime}}(\mathbf{q})$ from the correlators at the lowest simulated temperature $T=220 \mathrm{~K}$, which is close to $T_{c}^{M D}$. On the other hand we have used the static correlators from the simulation to solve Eq. (5) by iterations. This allows to locate the MCT-temperature $T_{c}^{M C T}$, at which the trivial solution $f_{\lambda \lambda^{\prime}}(\mathbf{q}) \equiv 0$ changes discontinuously to $f_{\lambda \lambda^{\prime}}(\mathbf{q}) \neq 0$.

As a result we have found

$$
T_{c}^{M C T} \cong 267 K
$$

The corresponding critical nonergodicity parameters from MCT are given together 


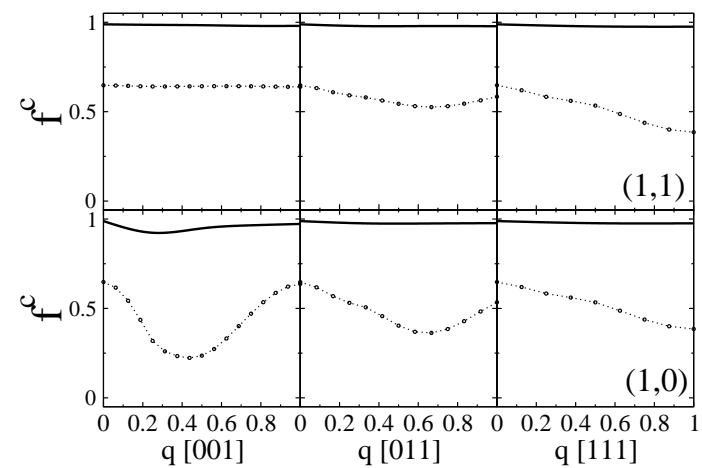

Fig. 8. q-dependence of the critical nonergodicity parameters along the [001], [011] and [111] directions for $\lambda=\lambda^{\prime}=(1,0),(1,1)$. The result from the MD simulation is given by the solid line and from MCT by the dots (the line connecting the dots are a guide for the eye)

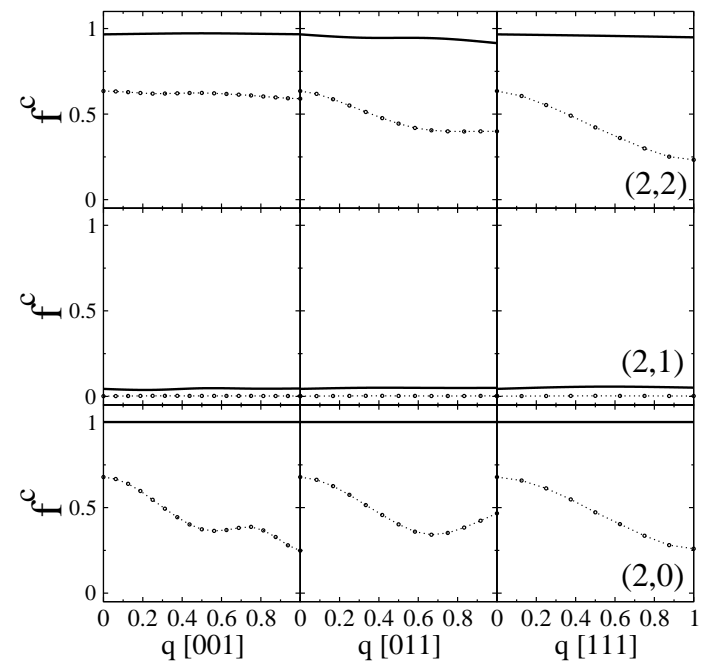

Fig. 9. Same as Fig. 8, but for $\lambda=\lambda^{\prime}=(2,0),(2,1),(2,2)$

with those from the simulation in Figures 8-10. The q-dependence is shown along three highly symmetric reciprocal directions. Let us discuss first $\ell=\ell^{\prime}=1$ (Fig. 8). The numerical result is very close to one for all q. For $m=m^{\prime}=0$, there is a shallow minimum along [001] direction. This minimum also appears in the corresponding MCT-result. However, $f_{10,10}^{c}(\mathbf{q})$ from MCT is not close to one and deviates from the MD-result by about 50 per cent. Furthermore, the former exhibits a much 


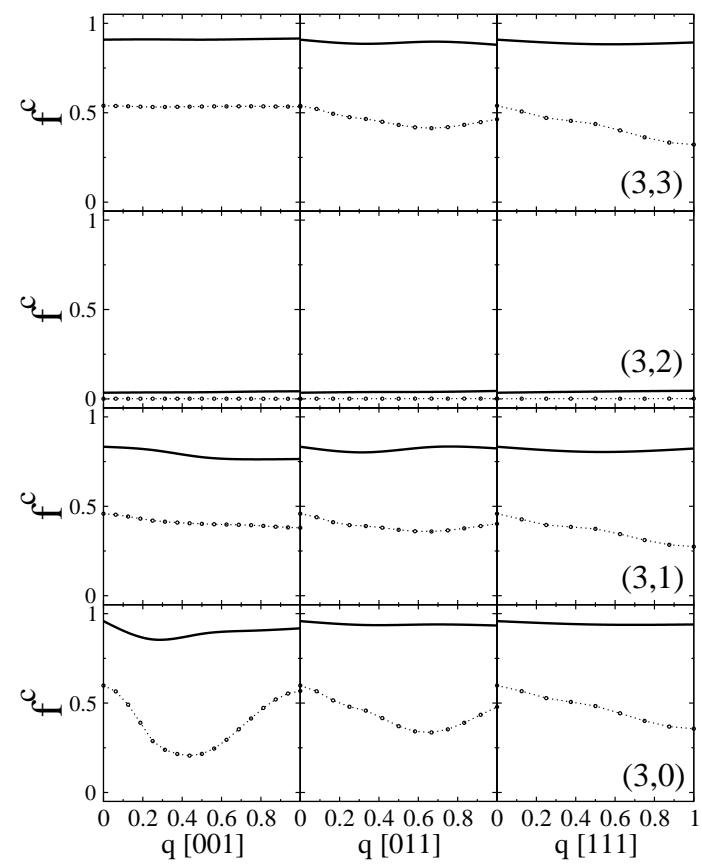

Fig. 10. Same as Fig. 8, but for $\lambda=\lambda^{\prime}=(3,0),(3,1),(3,2),(3,3)$

stronger q-dependence, whereas the MD-results are practically q-independent with exception for $m=m^{\prime}=0$ along the [001] direction. Similar conclusions can be drawn for $\ell=\ell^{\prime}=2$ (Fig. 9) and $\ell=\ell^{\prime}=3$ (Fig. 10). There are two features which are reproduced by MCT. First, we observe that the MCT-result for $f_{21,21}^{c}(\mathbf{0})$ (Fig. 9) and $f_{32,32}^{c}(\mathbf{0})$ (Fig. 10) is practically zero, in semi-quantitative agreement with the corresponding MD-result. This is even true for all q. Second, the hierarchy $f_{21,21}^{c}(\mathbf{0})<f_{22,22}^{c}(\mathbf{0})<f_{20,20}^{c}(\mathbf{0})$ and $f_{32,32}^{c}(\mathbf{0})<f_{31,31}^{c}(\mathbf{0})<f_{33,33}^{c}(\mathbf{0})<f_{30,30}^{c}(\mathbf{0})$ is valid for both MCT and MD.

\section{Summary and conclusions}

The main motivation of the present paper has been the test of the validity of the MCT predictions for the glassy behavior of molecular crystals. MCT has been recently extended from liquids to molecular crystals [17]. Since the corresponding equations of motion have the same structure as for multicomponent simple liquids, the MCT predictions for the liquid systems also hold for molecular crystals.

In order to check the validity of these predictions we have performed a MD sim- 
ulation for a simple model of chloroadamantane. In contrast to earlier simulations [22,23], we have used a rigid fcc-lattice, however, with an appropriately chosen temperature-dependent lattice constant $a(T)$. The decrease of $a(T)$ with decreasing temperature enhances the steric hindrance, which is responsible for the glassy dynamics. Figure 2 clearly demonstrates the validity of this physical picture.

There are two kinds of tests. On the one hand one can check the validity of the two scaling laws predicted by MCT [2,3,4]. Without attempting to calculate the corresponding exponents from first principles this represents an important qualitative test. On the other hand a first principle comparison can be made between the MD- and MCT-results, as has been done in Refs. [5,6,7,8,9,10,11,12,13,14,15]. Concerning the test of the scaling laws we have restricted ourselves to the second scaling law which holds for the $\alpha$-relaxation regime. In this regime, MCT for liquids and molecular crystals predicts the $t-T$ superposition principle which implies a data-collapse under rescaling time by the $\alpha$-relaxation time $\tau$. Figure 4 clearly demonstrates the validity of the second scaling law over a large temperature range for e.g. the collective and "self" correlators with $\lambda=\lambda^{\prime}=(1,0)$. Increasing $\ell$ and $\ell^{\prime}$ leads to a shrinking of that temperature range and also of the interval of validity for $t / \tau$ (see Fig. 5). The T-dependence of $\tau$ is shown in Figure 6. As predicted by MCT a power law dependence is found with a glass transition temperature $T_{c}^{M D} \cong 217 \mathrm{~K}$. Close to $T_{c}^{M D}$ deviations from the power law exist, due to ergodicity restoring processes. The value $T_{c}^{M D}$ is about 20 per cent below $T_{c}^{M C T}$, consistent with what has been found for liquids $[5,6,7,8,9,10,11]$.

The MD-results have shown a very high sensitivity of the relaxational behavior on $m$. For instance the collective correlators for $\ell=\ell^{\prime}=2$ and $m=m^{\prime}=0,2$ exhibit a two-step relaxation process with a plateau very close to one, but a very peculiar behavior for $m=m^{\prime}=1$ (see Fig.7a). For the latter there is practically no plateau. The same holds for $\ell=\ell^{\prime}=3$ and $m=m^{\prime}=2$ (see Fig. 7b). A first principle comparison between the MD- and MCT-results (see Figs. 8-10) reproduces this unusual $\mathrm{m}$-dependence. We stress that this unusually low plateau value is not related to a small corresponding static correlator. However, the critical nonergodicity parameters for $\ell=\ell=2, m=m^{\prime}=0,2$ and for $\ell=\ell^{\prime}=3$, $m=m^{\prime}=0,1,3$ from MCT deviate by about 50 per cent from the numerical ones. This strong discrepancy can have several reasons. First, the truncation at $\ell_{\max }=4$ may lead to a significant underestimation of the critical nonergodicity parameters. Second, there may be quite a different reason we want to explain now. The potential energy of the rigid molecular crystal can be written as follows:

$$
V\left(\Omega_{1}, \ldots, \Omega_{N}\right)=V_{0}+\sum_{n} V_{1}\left(\Omega_{n}\right)+\frac{1}{2} \sum_{n \neq m} V_{2}\left(\Omega_{n}, \Omega_{m}\right)
$$

in case of two-body interactions. The isotropic part $V_{0}$ is not essential. Besides the pair interactions given by $V_{2}$ there is a one-particle term $V_{1}$, which is a kind of crystal field, due to the crystal's anisotropy. $V_{1}(\Omega)$ will have a finite number of local 
minima. If the pair interaction $V_{2}$ would be zero, the molecules at finite temperatures would perform thermally activated jumps between these local minima, as can be really seen in Figure 2 of Ref. [22]. Starting in one of the local minima it is obvious that the long time limit of, e.g. the "self" correlators, will be positive. This happens for all initial conditions with energy below the corresponding energy barrier, and has already been investigated for a $\phi^{4}$-model [24]. This type of cage effect generated by an anisotropic one-particle potential is not accounted for by MCT which describes a two-, three-, etc. particle-cage-effect in a self consistent way. We believe that the large numerical values for the critical nonergodicity parameters are mainly due to such a one-particle-cage-effect.

Let us finally comment on the role of phonons, which are absent in the present MD-simulation, and also in MCT for molecular crystals [17]. A comparison of, e.g. $T_{c}^{M D}$ and $\gamma$ from the present simulation with earlier ones for the same model for chloroadamantane but on a non-rigid lattice shows that phonons do not have a noticeable influence on these quantities and on the long time dynamics.

\section{Acknowledgments}

Two of the authors (F. A. and M. D.) wish to acknowledge the use of the facilities of the IDRIS (Orsay, France) and the CRI (Villeneuve d'Ascq, France) where calculations were carried out. This work was supported by the INTERREG III (FEDER) program (Nord-Pas de Calais/Kent).

\section{References}

[1] U. Bengtzelius, W. Götze, and A. Sjölander, J. Phys. C17 (1984) 5915

[2] W. Götze and L. Sjögren, Rep. Prog. Phys. 55 (1992) 241

[3] W. Götze, J. Phys.: Condens. Matter 11, (1999) A1

[4] W. Kob, Les Houches lecture notes 2002, cond-mat/0212344

[5] M. Nauroth and W. Kob, Phys. Rev. E55 (1997) 657

[6] C. Theis and R. Schilling, J. Non-Cryst. Solids 235-237 (1998) 106

[7] A. Winkler, A. Latz, R. Schilling and C. Theis, Phys. Rev. E62 (2000) 8004

[8] L. Fabbian, A. Latz, R. Schilling, F. Sciortino, P. Tartaglia, and C. Theis, Phys. Rev. E60 (1999) 5768

[9] C. Theis, F. Sciortino, A. Latz, R. Schilling, and P. Tartaglia, Phys. Rev. E62 (2000) 1856

[10] F. Sciortino and W. Kob, Phys. Rev. Lett. 86 (2001) 648 
[11] S. H. Chong and F. Sciortino, Europhys. Lett. 64 (2003) 197

[12] W. Kob, M. Nauroth, and F. Sciortino, J. Non-Cryst. Solids 307-310 (2002) 181

[13] G. Foffi, W. Götze, F. Sciortino, P. Tartaglia, and Th. Voigtmann, Phys. Rev. E69 (2004) 011505

[14] Th. Voigtmann, Phys. Rev. E68, (2003) 051401

[15] Th. Voigtmann, A. M. Puertas, and M. Fuchs, Phys. Rev. E70 (2004) 061506

[16] H. Suga and S. Seki, J. Non-Cryst. Solids 16 (1974) 171

[17] M. Ricker and R. Schilling, Phys. Rev. E72 (2005) 011508

[18] M. Ricker and R. Schilling, Phys. Rev. E69 (2004) 061105

[19] M. Letz, R. Schilling, and A. Latz, Phys. Rev. E62 (2000) 5173

[20] F. Affouard, J.-F. Willart, and M. Descamps, J. Non-Cryst. Solids 307-310 (2002) 9

[21] M. Foulon, T. Belgrand, C. Gors, and M. More, Acta Cryst. B45 (1989) 404

[22] F. Affouard, E. Cochin, R. Decressain, and M. Descamps, Europhys. Lett. 53 (2001) 611

[23] F. Affouard and M. Descamps, Phys. Rev. Lett. 87 (2001) 035501

[24] W. Kob and R. Schilling, J. Phys.: Condens. Matter 3 (1991) 9195 